\title{
Skeletal muscle mass and sarcopenia can be determined with 1.5-T and 3-T neck MRI scans, in the event that no neck CT scan is performed
}

\author{
Aniek T. Zwart ${ }^{1,2,3}$ (D) Jan-Niklas Becker ${ }^{2} \cdot$ Maria J. Lamers $^{2} \cdot$ Rudi A. J. O. Dierckx ${ }^{2} \cdot$ Geertruida H. de Bock ${ }^{1} \cdot$ \\ Gyorgy B. Halmos ${ }^{3}$. Anouk van der Hoorn ${ }^{2}$
}

Received: 4 May 2020 / Revised: 17 August 2020 / Accepted: 22 October 2020 / Published online: 21 November 2020

(C) The Author(s) 2020

\begin{abstract}
Objectives Cross-sectional area (CSA) measurements of the neck musculature at the level of third cervical vertebra (C3) on CT scans are used to diagnose radiological sarcopenia, which is related to multiple adverse outcomes in head and neck cancer (HNC) patients. Alternatively, these assessments are performed with neck MRI, which has not been validated so far. For that, the objective was to evaluate whether skeletal muscle mass and sarcopenia can be assessed on neck MRI scans.

Methods HNC patients were included between November 2014 and November 2018 from a prospective data-biobank. CSAs of the neck musculature at the C3 level were measured on CT $(n=125)$ and MRI neck scans $(n=92$ on 1.5 -T, $n=33$ on 3-T). Measurements were converted into skeletal muscle index (SMI), and sarcopenia was defined $\left(\mathrm{SMI}<43.2 \mathrm{~cm}^{2} / \mathrm{m}^{2}\right)$. Pearson correlation coefficients, Bland-Altman plots, McNemar test, Cohen's kappa coefficients, and interclass correlation coefficients (ICCs) were estimated.

Results CT and MRI correlated highly on CSA and SMI $(r=0.958-0.998, p<0.001)$. The Bland-Altman plots showed a nihil mean $\Delta$ SMI $\left(-0.13-0.44 \mathrm{~cm}^{2} / \mathrm{m}^{2}\right)$. There was no significant difference between CT and MRI in diagnosing sarcopenia (McNemar, $p=0.5-1.0$ ). Agreement on sarcopenia diagnosis was good with $\kappa=0.956-0.978$ and $\kappa=0.870-0.933$, for 1.5 - T and 3-T respectively. Observer ICCs in MRI were excellent. In general, T2-weighted images had the best correlation and agreement with CT.

Conclusions Skeletal muscle mass and sarcopenia can interchangeably be assessed on CT and 1.5-T and 3-T MRI neck scans. This allows future clinical outcome assessment during treatment irrespective of used modality.

Key Points

- Screening for low amount of skeletal muscle mass is usually measured on neck CT scans and is highly clinical relevant as it is related to multiple adverse outcomes in head and neck cancer patients.

- We found that skeletal muscle mass and sarcopenia determined on CT and 1.5-T and 3-T MRI neck scans at the C3 level can be used interchangeably.

- When CT imaging of the neck is missing for skeletal muscle mass analysis, patients can be assessed with 1.5-T or 3-T neck MRIs.
\end{abstract}

Keywords Sarcopenia · Head and neck neoplasms · Muscle, skeletal · Tomography, X-ray computed · Magnetic resonance imaging

Aniek T. Zwart

a.t.zwart@umcg.nl

1 Department of Epidemiology, University Medical Center Groningen, 30.001, Hanzeplein 1, 9700 RB Groningen, The Netherlands
2 Department of Radiology, University Medical Center Groningen, Groningen, The Netherlands

3 Department of Otolaryngology and Head and Neck Surgery, University Medical Center Groningen, Groningen, The Netherlands 


$\begin{array}{ll}\text { Abbreviations } \\ \text { C3 } & \text { Third cervical vertebra } \\ \text { CSA } & \text { Cross-sectional area } \\ \text { HU } & \text { Hounsfield unit } \\ \text { L3 } & \text { Third lumbar vertebra } \\ \text { LSM } & \text { Left sternocleidomastoid muscle } \\ \text { PM } & \text { Paravertebral muscles } \\ \text { RSM } & \text { Right sternocleidomastoid muscle } \\ \text { SMI } & \text { Skeletal muscle index } \\ \text { SMM } & \text { Skeletal muscle mass }\end{array}$

\section{Introduction}

Head and neck cancers have a great impact as combined they are the sixth most common cancer in Europe, with more than 250,000 cases and 63,500 deaths annually [1]. In The Netherlands, incidence of patients with head and neck cancer has increased by 47\% between 1989 and 2019 [2]. Sarcopenia, or low skeletal muscle mass, is defined by the European Working Group on Sarcopenia in Older People (EWGSOP) as a progressive and generalized skeletal muscle disorder that is associated with increased likelihood of adverse outcomes [3]. Sarcopenia in head and neck cancer patients represents an important population burden with a prevalence of $6.6-70.9 \%$ and is related with frailty, post-operative complications, chemotherapy dose-limiting toxicity, and decreased overall survival and relapse-free survival [4-6]. Previous studies have indicated that females are more prone to sarcopenia than males $[4,7]$.

According to the EWGSOP, presence of low skeletal muscle mass (SMM) confirms the diagnosis of sarcopenia [3]. Singleslice assessment of SMM on neck CT scans at the level of the third cervical vertebra (C3) has recently been validated by Swartz et al [8] and could therefore be a breakthrough for a novel radiological biomarker in head and neck cancer patients. However, certain patients with head and neck cancer subtypes will not undergo $\mathrm{CT}$ imaging of the neck, but instead undergo imaging with the use of magnetic resonance imaging (MRI). This is especially the case not only for nasopharyngeal and sinonasal cancer, but also for other malignancies situated cranial to the hyoid bone [9-12], and in patients with iodinated contrast allergies.

Previous studies indicated that cross-sectional area (CSA) measurements on CT and MRI scans can be used interchangeably for muscles of the limbs [13], thigh [14], paraspinal skeletal back muscles $[15,16]$, and abdomen [17]. A recently published retrospective study indicates that CSA measurements of the neck musculature at the $\mathrm{C} 3$ level, made on CT and MRI scans, can also be used interchangeably [18], and additionally CSA measurements made on CT scans at the level of C3 are reproducible [19]. However, it has not yet been clarified if CSA measurements at the level of $\mathrm{C} 3$ are comparable for all MRI sequences and field strengths in relation to CT. It is also unknown if CSA measurements of the neck musculature on MRI at the $\mathrm{C} 3$ level are robust in context of reproducibility and repeatability. We hypothesize that SMM measurements on CT and MRI neck scans at the level of $\mathrm{C} 3$ are equivalent. Hence, our aim is to analyze the agreement and correlation of CSA measurements made on CT and various MRI neck scans made with different sequences and flied strengths at the level of $\mathrm{C} 3$.

\section{Materials and methods}

Data was derived from OncoLifeS, a large oncological databiobank [20]. Patients were prospectively included after written informed consent, and imaging data was retrospectively analyzed. The Medical Ethical Committee of the University Medical Centre of Groningen approved the data-biobank and the use of the data.

\section{Patients and study design}

Between November 2014 and November 2018, 1221 consecutive patients diagnosed with stage I-IV squamous cell carcinoma of the oral cavity, larynx, oropharynx, hypopharynx, and nasopharynx in the University Medical Centre of Groningen gave their informed consent in using their data in OncoLifeS (Fig. 1). In the presented analyses, patients were

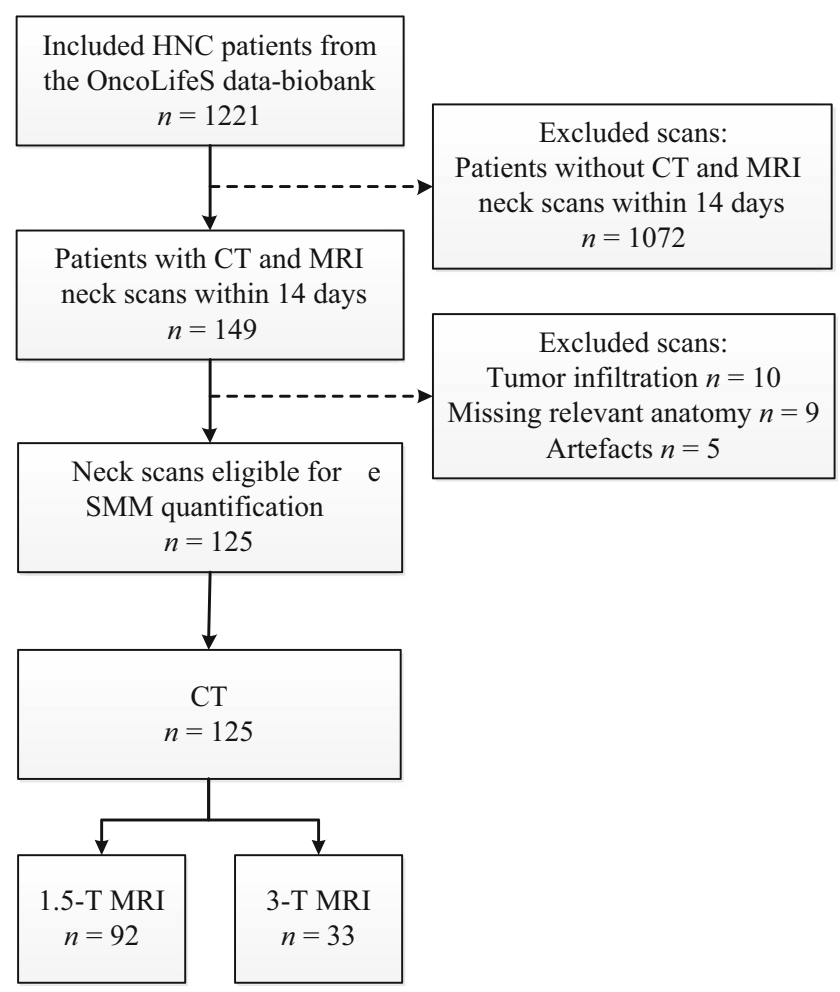

Fig. 1 Flowchart of included and excluded patients. $H N C$ head and neck cancer, $M R I$ magnetic resonance imaging, $C T$ computed tomography, $S M M$ Skeletal muscle mass 
included with a maximum interval of 14 days between CT and MRI scans, to minimize time-dependent pathophysiological changes $(n=149)$. Excluded were scans with lymph node invasion into relevant muscles $(n=10)$, scans not capturing the relevant anatomy $(n=9)$, or scans with motion artefacts $(n=5)$. Total sample size was 125 patients with a mean age of $63(42-82)$ years. Mean age for males was $64(42-82)$ years and mean age for females was 61 (42-81) years.

\section{Image acquisition}

All pre-treatment scans were acquired for clinical purposes and performed on a Siemens Healthcare CT (Biograph64, SOMATOM Force, SOMATOM Open, SOMATOM Definition AS or SOMATOM Definition Flash) and MRI scanners (1.5-T Area or 3-T Prisma or Skyra). CT scans were performed with intravenous iodine contrast $(n=115)$ or without $(n=10)$. CT image preference was a soft tissue kernel nearest to $30(30, n=78 ; 40, n=39 ; 26, n=8)$ with a slice thickness of $2.00 \mathrm{~mm}(2.00 \mathrm{~mm}, n=107 ; 1.00 \mathrm{~mm}, n=18)$.

Available sequences on 1.5-T MRI were T1 without contrast (repetition time/echo time (TR/TE) 2210-2780/55 ms, flip angle $150^{\circ}$, matrix $256 \times 256$, slice thickness $3 \mathrm{~mm}$, and spacing between slices $3.6 \mathrm{~mm}$ ), T1 vibe (volumetric interpolated breath-hold examination) with fat suppression and gadolinium 10-22 ml (TR/TE $5.04 / 2.34 \mathrm{~ms}$, flip angle $10^{\circ}$, matrix $224 \times 224$, slice thickness $0.9 \mathrm{~mm}, 3 \mathrm{D}$ acquisition), and T2 (TR/ TE 5990-8940/81 ms, flip angle $129-149^{\circ}$, matrix $320 \times 320$, slice thickness $3 \mathrm{~mm}$, spacing between slices $3.6 \mathrm{~mm}$ ).

For 3-T, the sequences used were T1 turbo spin echo (TSE) without contrast and T1 TSE with gadolinium 11-21 ml (both TR/TE $893-1020 / 11 \mathrm{~ms}$, flip angle $139-160^{\circ}$, matrix $640 \times$ 640 , slice thickness $3 \mathrm{~mm}$, spacing between slices $3.6 \mathrm{~mm}$ ), T1 vibe 3D-DIXON with fat suppression and gadolinium 11-21 ml (TR/TE 4.49-5.5/2.46 ms, flip angle $9^{\circ}$, matrix 256-264 × 256-264, slice thickness $0.9 \mathrm{~mm}$ ), and T2 TSE DIXON (TR/TE 5460-6880/77-97 ms, flip angle 120$126^{\circ}$, matrix $640 \times 640$, slice thickness $3 \mathrm{~mm}$, spacing between slices $3.6 \mathrm{~mm}$ ).

\section{Skeletal muscle image analysis}

SMM quantification was conducted with the Aquarius workstation iNtuition edition program (v.4.4.13.P6, TeraRecon, Inc.). Slice selection was performed according the validated procedure of Swartz et al [8]. However, MRI scans had a relatively large pitch and slice thickness. If a fully closed arch could not be identified, the most caudal slice was chosen where the posterior arch was nearest to a closed arch (Fig. 2). Interpolation between images was utilized, and angulation was prohibited to ensure reproducibility.

CT measurements were performed as described earlier [4] (Fig. 2a). Delineation made on the MRI sequences was done manually, eyeballing the relevant structures including the paravertebral muscles and both sternocleidomastoid muscles. Total CSA on MRI corresponded with the true delineated area.

\section{Observer reliability}

All measurements were performed by JNB (obs. 1; medical student) after an extensive training of 2 weeks under the supervision of ATZ and AvdH (board-certified neuro/head-neck radiologist with 3 years of experience with these specific measurements). Interobserver reliability was analyzed with intraclass correlation coefficients. Interobserver analysis was performed in a randomly reselected sample $(n=25)$ on 1.5-T neck MRI scans by ATZ (obs. 2; PhD student with a background in medicine and as a radiologic technician, with 3 years of experience with the used acquisition program) and MJL (obs. 3 ; with 7 years of experience as a board-certified neuro/headneck radiologist, without specific training needed). Level of slice selection was also analyzed per observer and measurement data were blinded for the observers. Furthermore, intraobserver analysis was done with a time interval of $>2$ weeks between the first and the second CSA measurements $(*)$.

\section{Sarcopenia diagnosis}

Skeletal muscle index $\left(\mathrm{SMI}, \mathrm{cm}^{2} / \mathrm{m}^{2}\right)$ at the level of L3 was determined, which is considered a surrogate marker for the total body SMM [21]. First, CSA at C3 $\left(\mathrm{cm}^{2}\right)$ was converted to CSA at $\mathrm{L} 3\left(\mathrm{~cm}^{2}\right)$ according the algorithm of Swartz et al [8] (see Eq. 1). Second, calculated CSA at L3 was furthermore adjusted for patient height $\left(\mathrm{m}^{2}\right)$ resulting in SMI (see Eq. 2). The outcome, or SMM status, was presented continuously with SMI, and dichotomously as (non-)sarcopenic based on previously published SMI cut-off value $\left(<43.2 \mathrm{~cm}^{2} / \mathrm{m}^{2}\right)$ [7]

$$
\begin{gathered}
\text { CSA at L3 } \left.\left(\mathrm{cm}^{2}\right)=27.304+1.363 * \text { CSA at C3 }\left(\mathrm{cm}^{2}\right)-0.671 * \text { Age (years }\right) \\
+0.640 * \text { Weight }(\mathrm{kg})+26.442 * \operatorname{Sex}(1=\text { Female }, 2=\text { Male })
\end{gathered}
$$

SMI $\left(\mathrm{cm}^{2} / \mathrm{m}^{2}\right)=$ CSA at L3 $\left(\mathrm{cm}^{2}\right) /$ Height $\left(\mathrm{m}^{2}\right)$

\section{Statistical analysis}

Continuous data was analyzed for normality with the ShapiroWilk test (normality: $\alpha>0.05$ ) and Q-Q plots. The baseline characteristics of the patient cohort were summarized. Differences of baseline characteristics between patients in the 1.5-T group and those in the 3-T group were analyzed with independent $t$ tests and Pearson's chi-square tests. CSA measurements made with $\mathrm{CT}$ and MRI were analyzed with Pearson's correlation coefficient. CT was selected as the reference standard, as previously described algorithm was generated on neck CTs. Bland-Altman plots with $95 \%$ confidence 
Fig. 2 Skeletal muscle measurements at the level of $\mathrm{C} 3$ on axial neck $\mathrm{CT}$ and MRI images. (1) CSA of the right sternocleidomastoids muscle, (2) CSA of the left sternocleidomastoids muscle, and (3) CSA of the paravertebral muscles. a Acquired using CT. The green area represents the delineated muscle area which corresponds with the preset Hounsfield units range of -29 until 150. Other densities are automatically excluded from the measured area. $\mathbf{b}-\mathbf{d}$

Slices acquired using 1.5-T MRI. The before-mentioned muscles are delineated red with (b) T2 without fat suppression, (c) T1 with contrast and fat suppression, and (d) being T1 sequence without contrast and without fat suppression. $C 3$ third cervical vertebra, $C T$ computed tomography, MRI magnetic resonance imaging, CSA crosssectional area
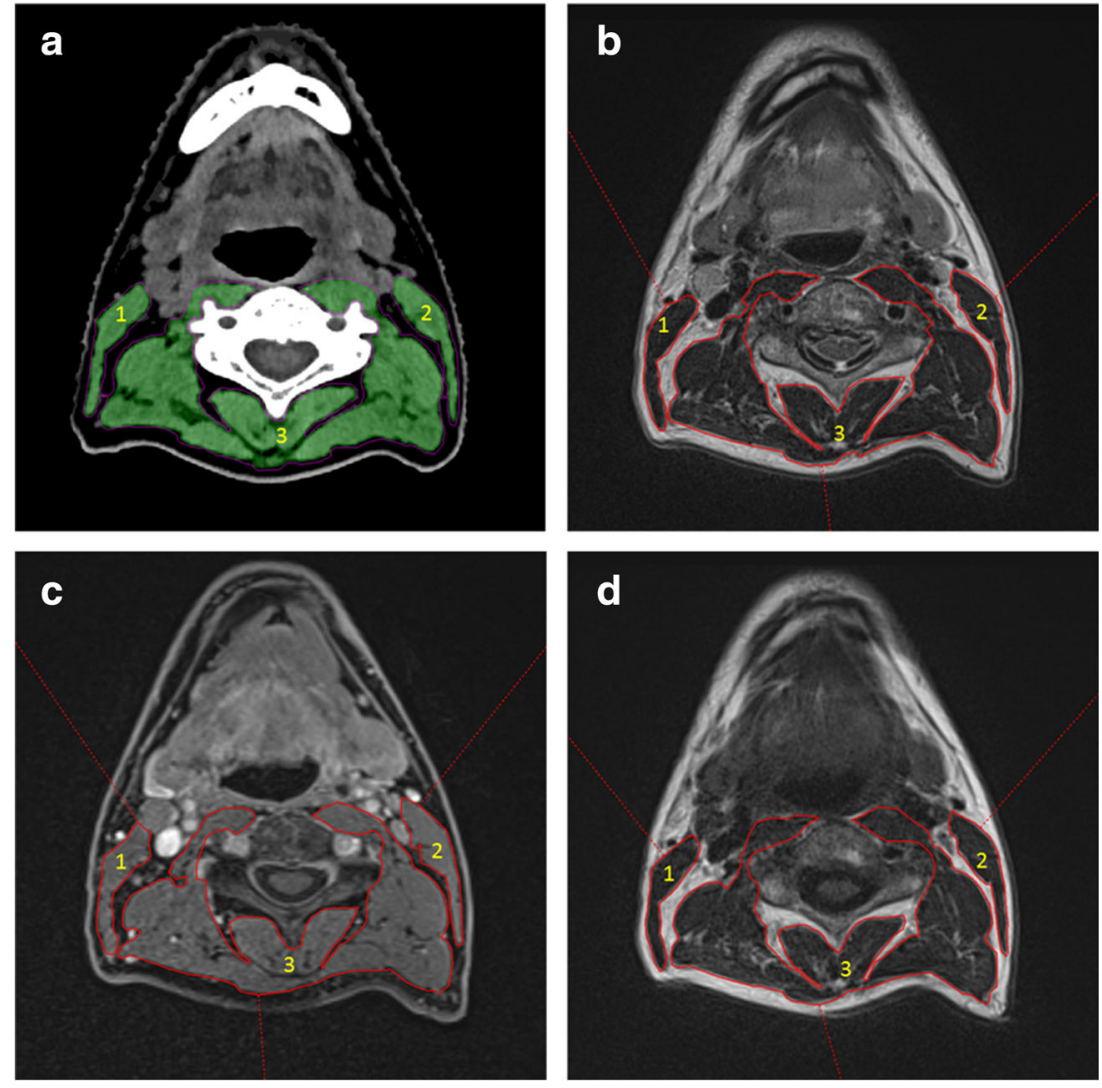

intervals were created with mean SMI (CT + MRI/2) and $\triangle \mathrm{SMI}(\mathrm{CT}-\mathrm{MRI})$ to visualize agreement, possible biases, or outliers. A linear regression was performed and added to the Bland-Altman plot when significant. Interclass correlation coefficients (ICCs) were performed to analyze observer reliability. Data was furthermore stratified for gender, as gender differences were previously observed $[4,7]$. To estimate clinical relevance, difference and agreement of sarcopenia diagnosis between CT and MRI were analyzed with the McNemar test and Cohen's kappa coefficient $(\kappa)$ respectively. A nonsignificant McNemar test corresponds with no difference in diagnosis between the two modalities [22], and a $\kappa>0.81$ was considered perfect agreement [23]. Variables were statistically significant if $\alpha<0.05$. There was no missing data. SPSS version 23.0 was used for statistical analysis.

\section{Results}

\section{Patient and disease characteristics}

The intended sample size consisted of 125 head and neck cancer patients with pre-treatment CT and MRI scans (see Table 1 for baseline characteristics). The majority of the patient sample was male (72\%), and the mean age at time of diagnosis was $63( \pm 9)$ years. Most patients had oropharyngeal cancer (48\%), followed by laryngeal (36\%), hypopharyngeal (14\%), oral (1\%), and nasopharyngeal cancer (1\%). Three quarters of patients had stage IIIIV advanced disease. None of the baseline variables showed significant differences $(p=0.053-0.84)$ between 1.5-T and 3-T MRI scans. All variables (characteristics as well outcomes) were normally distributed (Shapiro-Wilk: $p>0.05)$.

\section{Correlation and agreement between $\mathrm{CT}$ and MRI}

Mean time between CT and MRI was $3.27 \pm 3.42$ days for 1.5-T scans and $1.94 \pm 2.76$ days for 3-T scans. The scores for CSAs and SMI as measured with CT and MRI for both 1.5 - $\mathrm{T}$ and 3-T were highly correlated $(r=0.958-0.997$, $p<0.001$ ) (Table 2). Some minor differences were observed when analyzing the three delineated structures separately, as the paravertebral muscles scored relatively the highest correlation ( $r=0.988-0.995)$ compared to the left of the right sternocleidomastoid muscle $(r=0.958-0.976$ and $r=0.961-$ 0.986 respectively). T2 DIXON sequence on the 3-T MRI scanner had marginally the highest correlation based on total CSA and SMI $(r=0.998)$.

When visualizing the agreement between the 1.5-T MRI scans and CT, a few outliers $(n=5-6)$ could be identified per sequence (Fig. 3). Noticeably, almost all outliers with the high 
Table 1 Characteristics of included patients

\begin{tabular}{lccc}
\hline & $1.5-\mathrm{T}(n=92)$ & $3-\mathrm{T}(n=33)$ & $p$ value \\
\hline Sex & & & $0.31^{\mathrm{a}}$ \\
Female & $28(30.4 \%)$ & $7(21.2 \%)$ & \\
Male & $64(69.6 \%)$ & $26(78.8 \%)$ & \\
Age (years) & $62.7( \pm 9.0)$ & $64.4( \pm 10.3)$ & $0.37^{\mathrm{b}}$ \\
BMI (kg/m $\left.{ }^{2}\right)$ & $25.0( \pm 5.3)$ & $25.2( \pm 4.0)$ & $0.82^{\mathrm{b}}$ \\
Tumour site & & & $0.13^{\mathrm{a}}$ \\
Oropharynx & $48(52.2 \%)$ & $12(36.4 \%)$ & \\
Larynx & $28(30.4 \%)$ & $17(51.5 \%)$ & \\
Hypopharynx & $15(16.3 \%)$ & $3(9.1 \%)$ & \\
Oral cavity or nasopharynx & $1(1.1 \%)$ & $1(3.0 \%)$ & \\
T-classification* & & & $0.63^{\mathrm{b}}$ \\
T1 & $12(13.0 \%)$ & $2(6.1 \%)$ & \\
T2 & $23(25.0 \%)$ & $7(21.2 \%)$ & \\
T3 & $31(33.7 \%)$ & $14(42.4 \%)$ & \\
T4 & $26(28.3 \%)$ & $10(30.3 \%)$ & \\
N-classification* & & & $0.053^{\mathrm{a}}$ \\
N0 & $25(37.2 \%)$ & $15(45.5 \%)$ & \\
N+ & $67(62.8 \%)$ & $18(54.5 \%)$ & \\
Oncologic stage* & & \multicolumn{2}{c}{$0.84^{\mathrm{a}}$} \\
I-II & $22(2.2 \%)$ & $4(12.1 \%)$ & \\
III & $61(66.3 \%)$ & $22(60.6 \%)$ & \\
IV & & & \\
\hline & & & \\
\hline
\end{tabular}

Patients stratified according to MRI field strength $1.5-\mathrm{T}$ and 3-T. Categorical data is given with percentage of total group size $n$. Continues data is given as mean with standard deviation. Significance $p$ calculated by ${ }^{\mathrm{a}}$ Pearson's chi-square test and ${ }^{\mathrm{b}}$ Student's independent $t$ test. *Staging confirmed with the 7 th edition of the American Joint Committee on Cancer Manual

Table 2 Correlation coefficients and sarcopenia categorization between $\mathrm{CT}$ and MRI sequences

\begin{tabular}{llllll}
\hline & Total CSA & RSM & LSM & PM & SMI \\
\hline 1.5-T & & & & & \\
T1 & $0.987^{*}$ & $0.969^{*}$ & $0.966^{*}$ & $0.988^{*}$ & $0.997^{*}$ \\
T1 vibe & $0.989^{*}$ & $0.961^{*}$ & $0.958^{*}$ & $0.989^{*}$ & $0.997^{*}$ \\
T2 & $0.988^{*}$ & $0.971^{*}$ & $0.959^{*}$ & $0.988^{*}$ & $0.997^{*}$ \\
3-T & & & & & \\
T1 & $0.990^{*}$ & $0.986^{*}$ & $0.976^{*}$ & $0.991^{*}$ & $0.997^{*}$ \\
T1 + contrast & $0.989 *$ & $0.967^{*}$ & $0.970^{*}$ & $0.991^{*}$ & $0.997 *$ \\
T1 DIXON & $0.990^{*}$ & $0.980^{*}$ & $0.976^{*}$ & $0.990^{*}$ & $0.997 *$ \\
T2 DIXON & $0.994^{*}$ & $0.965^{*}$ & $0.962^{*}$ & $0.995^{*}$ & $0.998^{*}$ \\
\hline
\end{tabular}

Comparing CT with 1.5-T $(n=92)$ and 3-T $(n=33)$ MRI sequences with Pearson's correlation coefficients for area measurements and the skeletal muscle index. $C T$ computed tomography, MRI magnetic resonance imaging, CSA cross-sectional area, RSM right sternocleidomastoid muscle, LSM left sternocleidomastoid muscle, PM paravertebral muscle, SMI skeletal muscle index, Sarc. sarcopenic. *Significant $p$ value $<0.001$ mean SMI region were found under the $95 \% \mathrm{CI}$ and vice versa. This trend was also illustrated when adding the regression line, which had a downward slope of -0.03 showing a small proportional bias. Mean $\Delta$ SMI was almost zero for all sequences, with the lowest delta of $0.01 \mathrm{~cm}^{2} / \mathrm{m}^{2}$ for the CT and T2 sequence, and the highest delta of $0.21 \mathrm{~cm}^{2} / \mathrm{m}^{2}$ for the $\mathrm{CT}$ and $\mathrm{T} 1$ sequence.

Bland-Altman plots between CT and the four 3-T MRI sequences were very similar to the 1.5 - $\mathrm{T}$ sequences (see Fig. 1 in supplementary results), with minimal mean $\triangle$ SMI. T2 DIXON was superior with the lowest mean $\Delta$ SMI of $0.17 \mathrm{~cm}^{2} / \mathrm{m}^{2}$.

\section{Observer agreement of CSA measurements in MRI}

Analysis on slice selection (Table 3 ) gave similar results for $\mathrm{T} 1$ and T2; in 56-64\% of the cases, the same slice was selected and the deviation was solely one slice. Selecting the same level was harder for T1 vibe, as 16-24\% of the cases the same slice was selected, and more than half of the cases had a deviation of 2 or more slices.

Excellent and significant intra- and interobserver reliability were achieved in the randomly reselected patients from the 1.5-T MRI group (Table 3). The intraobserver ICCs for total CSA and CSA of PM at the level of C3 were the highest $(r=0.997-0.998, p<0.001)$, independently of sequence, and T1 has the best interobserver ICC $(r=0.996$, $p<0.001)$. However, differences of ICCs between the three 1.5-T MRI sequences made were all marginal.

\section{Agreement in SMI in CT and MRI stratified for gender}

Subsequently, the mean $\Delta \mathrm{SMI}$ between $\mathrm{CT}$ and the three 1.5-T sequences were stratified into men $(n=64)$ and women $(n=28)$ in Bland-Altman plots (Fig. 4, for T1 and T2). For men, the mean $\Delta$ SMI was $0.11 \mathrm{~cm}^{2} / \mathrm{m}^{2}$ for both $\mathrm{T} 1$ and $\mathrm{T} 2$ and $0.23 \mathrm{~cm}^{2} / \mathrm{m}^{2}$ for $\mathrm{T} 1$ vibe. Here, the regression line was significant for T1 vibe $(p=0.037)$ but not for T1 $(p=$ $0.051)$ and $\mathrm{T} 2(p=0.156)$.

For women, the mean $\Delta$ SMI was the closest to zero for T1 vibe with $0.1 \mathrm{~cm}^{2} / \mathrm{m}^{2}\left(0.4 \mathrm{~cm}^{2} / \mathrm{m}^{2}\right.$ for $\mathrm{T} 1$ and $0.3 \mathrm{~cm}^{2} /$ $\mathrm{m}^{2}$ for T2). For women, linear regressions were all significant $(p<0.001)$ and displayed a proportional bias with a downward slope of -0.07 , which was relatively higher than in men $(-0.03-0)$, but remained a minor difference.

\section{Effect of use of CT or MRI on presence of sarcopenia diagnosis}

Categorization of sarcopenic patients with the different MRI field strengths compared to CT is shown in Table 4. With 1.5T MRI sequences, one $(+2 \%$ : T2) or two $(+4 \%$ : T1) additional patients were categorized as sarcopenic $(\mathrm{SMI}<$ 

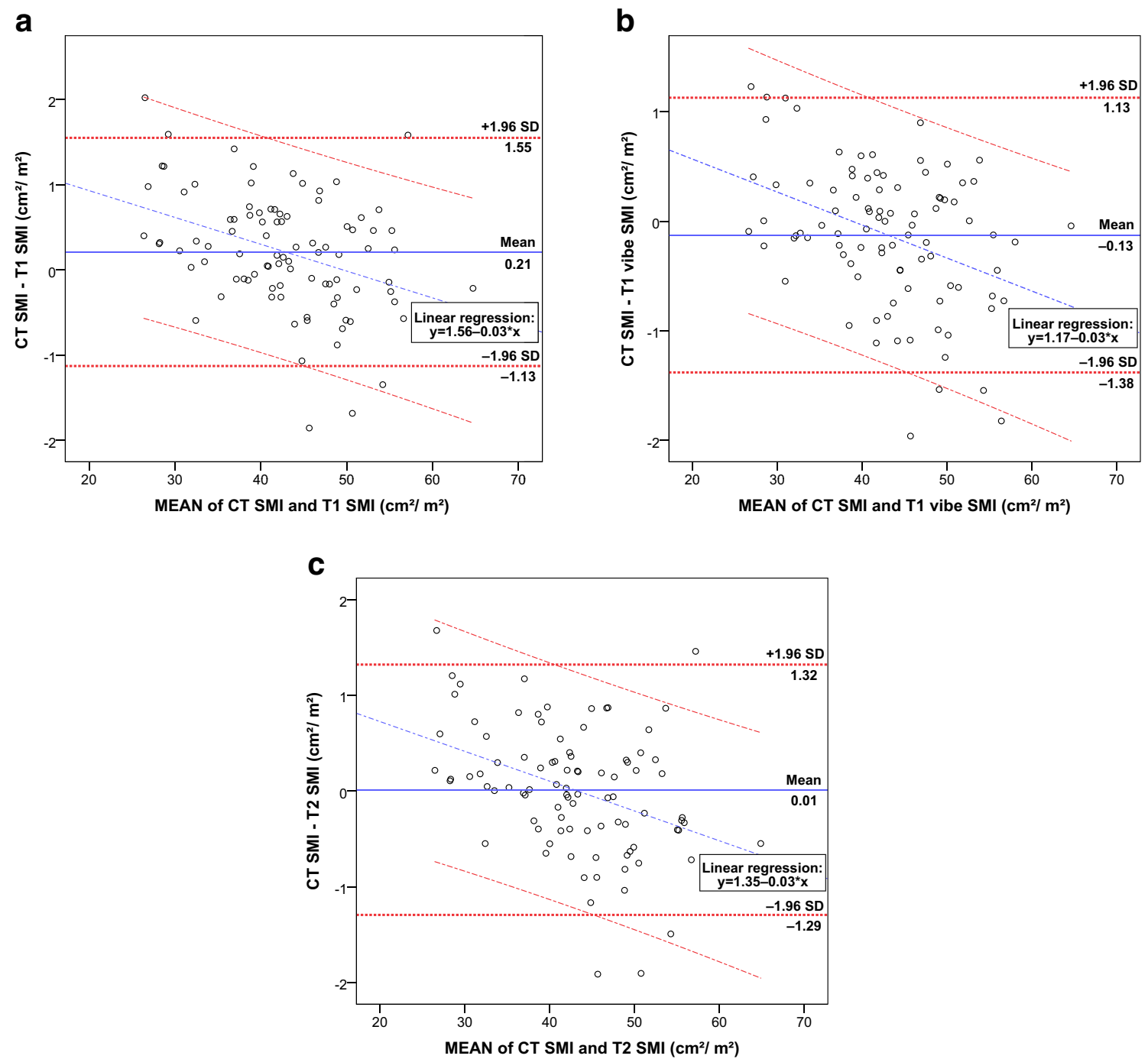

Fig. 3 Bland-Altman plots with mean SMI and $\Delta$ SMI between CT and 1.5-T MRI. Boundaries with the $95 \%$ confidence interval $( \pm 1.96$ times the standard deviation) are given for the mean $\Delta$ SMI and linear regression analysis. For all patients with a 1.5 -T MRI $(n=92)$ comparing CT SMI versus T1 SMI (a), CT SMI versus T1 vibe SMI (b), and CT SMI versus T2 SMI (c). SMI skeletal muscle index, $C T$ computed tomography, $M R I$ magnetic resonance imaging

\section{Discussion}

$43.2 \mathrm{~cm}^{2} / \mathrm{m}^{2}$ ), except for T1 vibe as one patient was not categorized as sarcopenic $(-2 \%)$. Agreement of CT-derived SMI was almost perfect for all 1.5-T sequences $(\kappa \geq 0.956)$.

One (+ 9\%: T2 DIXON) or two (+18\%: T1, T1 contrast, and T1 DIXON) additional categorized sarcopenic patients were also seen in measurements made on 3-T sequences. The three $\mathrm{T} 1$ sequences had therefore relatively a lower agreement $(\kappa=0.870)$ in contrast to T2 DIXON $(\kappa=$ $0.933)$, nevertheless still both good. These differences were only found in men, as all women were identified as sarcopenic by all methods using the before mentioned cutoff value. The exact McNemar test using a binominal distribution showed no significance in sarcopenia categorization between $\mathrm{CT}$ and the examined MRI sequences $(p \geq 0.50)$.
This is the first study with CSA data on the level of C3 generated on MRI with different field strengths and sequences, in relation to $\mathrm{CT}$. We found an excellent correlation between CSA measurements on CT and 1.5-T and 3-T MRI neck scans at the level of C3. Differences in SMI (mean $\Delta$ SMI) between $\mathrm{CT}$ and MRI were minimal with good agreement and less than $5 \%$ outliers outside the $95 \%$ CI. Never previously published observer analysis for MRI in this context showed that slice selection was the easiest for $\mathrm{T} 1$ and $\mathrm{T} 2$ sequences in the 1.5-T group, and CSA measurements are both reliable and reproducible, as we found excellent intra- and interobserver ICCs. Agreement of CT and MRI was practically the same for men and women. Equivalent diagnosis of sarcopenia was made 
Table 3 Inter- and intraobserver reliability and slice selection

\begin{tabular}{|c|c|c|c|c|c|c|c|c|c|}
\hline \multicolumn{7}{|c|}{ Interobserver correlation with slice selection } & \multicolumn{3}{|c|}{ Intraobserver correlation } \\
\hline & CSA & Slice & Obs. 1 & Obs. 2 & Obs. 3 & ICCs $(95 \%$ CI $)$ & First & Second & ICCs $(95 \%$ CI) \\
\hline \multirow[t]{6}{*}{$\mathrm{T} 1$} & Total & & $38.95( \pm 9.11)$ & $39.40( \pm 9.78)$ & $39.11( \pm 10.10)$ & $0.996(0.993-0.998)^{*}$ & $39.07( \pm 9.00)$ & $38.95( \pm 9.11)$ & $0.998(0.994-0.999)^{*}$ \\
\hline & $\mathrm{PM}$ & & $32.59( \pm 7.66)$ & $32.86( \pm 8.18)$ & $32.64( \pm 8.54)$ & $0.996(0.992-0.998)^{*}$ & $32.61( \pm 7.54)$ & $32.59( \pm 7.66)$ & $0.997(0.993-0.999)^{*}$ \\
\hline & RSM & & $3.17( \pm 0.85)$ & $3.27( \pm 0.92)$ & $3.24( \pm 0.91)$ & $0.993(0.986-0.997) *$ & $3.20( \pm 0.83)$ & $3.17( \pm 0.85)$ & $0.992(0.981-0.996)^{*}$ \\
\hline & LSM & & $3.19( \pm 0.81)$ & $3.27( \pm 0.90)$ & $3.22( \pm 0.89)$ & $0.994(0.988-0.997)^{*}$ & $3.26( \pm 0.83)$ & $3.19( \pm 0.81)$ & $0.985(0.958-0.994)^{*}$ \\
\hline & & Same & Ref. & $16(64 \%)$ & $16(64 \%)$ & & & & \\
\hline & & 1 & Ref. & $9(36 \%)$ & $9(36 \%)$ & & & & \\
\hline \multirow[t]{7}{*}{ T1 vibe } & Total & & $39.51( \pm 9.16)$ & $6(24 \%)$ & $4(16 \%)$ & $0.993(0.983-0.997)^{*}$ & $39.72( \pm 9.14)$ & $39.51( \pm 9.16)$ & $0.997(0.993-0.999)^{*}$ \\
\hline & $\mathrm{PM}$ & & $33.14( \pm 7.67)$ & $33.86( \pm 8.88)$ & $33.32( \pm 8.58)$ & $0.993(0.987-0.997)^{*}$ & $33.16( \pm 7.63)$ & $33.14( \pm 7.67)$ & $0.998(0.995-0.999)^{*}$ \\
\hline & RSM & & $3.19( \pm 0.90)$ & $3.57( \pm 0.96)$ & $3.27( \pm 0.97)$ & $0.973(0.877-0.991)^{*}$ & $3.25( \pm 0.87)$ & $3.19( \pm 0.90)$ & $0.975(0.944-0.989)^{*}$ \\
\hline & LSM & & $3.18( \pm 0.81)$ & $3.50( \pm 0.96)$ & $3.23( \pm 0.90)$ & $0.968(0.905-0.987)^{*}$ & $3.31( \pm 0.85)$ & $3.18( \pm 0.81)$ & $0.961(0.870-0.985)^{*}$ \\
\hline & & Same & Ref. & $6(24 \%)$ & $4(16 \%)$ & & & & \\
\hline & & 1 & Ref. & $5(20 \%)$ & $8(32 \%)$ & & & & \\
\hline & & $\geq 2$ & Ref. & $14(56 \%)$ & $13(52 \%)$ & & & & \\
\hline \multirow[t]{6}{*}{$\mathrm{T} 2$} & Total & & $39.37( \pm 9.17)$ & $40.30( \pm 9.94)$ & $39.67( \pm 9.94)$ & $0.995(0.990-0.998)^{*}$ & $39.51( \pm 9.09)$ & $39.37( \pm 9.17)$ & $0.998(0.996-0.999) *$ \\
\hline & PM & & $32.87( \pm 7.67)$ & $33.60( \pm 8.40)$ & $33.23( \pm 8.43)$ & $0.994(0.989-0.997)^{*}$ & $32.95( \pm 7.61)$ & $38.89( \pm 7.67)$ & $0.998(0.996-0.999) *$ \\
\hline & RSM & & $3.25( \pm 0.86)$ & $3.39( \pm 0.91)$ & $3.24( \pm 0.89)$ & $0.991(0.997-0.996)^{*}$ & $3.28( \pm 0.85)$ & $3.25( \pm 0.86)$ & $0.992(0.982-0.996)^{*}$ \\
\hline & LSM & & $3.22( \pm 0.85)$ & $3.31( \pm 0.86)$ & $3.21( \pm 0.85)$ & $0.995(0.987-0.998)^{*}$ & $3.28( \pm 0.83)$ & $3.22( \pm 0.85)$ & $0.986(0.965-0.994) *$ \\
\hline & & Same & Ref. & $16(64 \%)$ & $14(56 \%)$ & & & & \\
\hline & & 1 & Ref. & $9(36 \%)$ & $11(44 \%)$ & & & & \\
\hline
\end{tabular}

ICCs for inter- and intraobserver analyses of skeletal muscle area measurements made by all three observers, and relative difference of slice selection between first observer and other observers $n=25$. CSA cross-sectional area, $C I$ confidence interval, ICCs intraclass correlation coefficients, $P M$ paravertebral muscles, $R S M$ right sternocleidomastoid muscle, $L S M$ left sternocleidomastoid muscle. *Significant $p$ value $<0.001$

with both modalities. However, preference could be made to utilize T2-weighted images as highest intra- and interobserver agreements, correlation, and agreements on Bland-Altman plots were achieved.

High correlations and agreement of CSA measurements of muscles on $\mathrm{CT}$ and MRI are universally found in previous articles [13-18]. We found, irrespective of the sequence or field strength, an excellent correlation for total CSA in the neck scans. Our correlation is higher than previous found for neck muscles at C3 [18], paraspinal back muscles [15], and abdominal muscles at L1 [17]. As Chargi et al [18] did not specify MRI acquisition parameters, it is hard to compare results. The study of Sinelnikov et al [17] using thresholdbased region growing segmentation also differs from this study and therefore hampers a direct comparison. Nevertheless, they found the highest correlation and agreement in T2-weighted images [17], which is in line with our present study. 1.5-T and 3-T MRI also showed previous excellent agreement in a small animal study comparing histology and CSA measures of different muscles [24]. The found small proportional bias might be based on the different methods used for CT and MRI delineation of CSA, which is similar to other studies that compared manual and threshold-based techniques in CSA measurements $[25,26]$. This finding was more emphasized in women probably due to relatively lower SMI ratios. Nevertheless, the agreement using the 95\% CI identified only a few outliers in our study. Bril et al [19] also found a significantly excellent interobserver agreement in CSA measurements on neck CT scans at the level of C3. Similar to our study, the highest ICCs in MRI were found in CSA of paravertebral muscles and total CSA at $\mathrm{C} 3$, and lowest ICCs in CSA of sternocleidomastoid muscle independently. Our results exceeded the intra- and interobserver ICCs in the study of Sinelnikov et al and Khil et al on abdominal scans $[16,17]$, possibly due to different delineation methods used for MRI in their studies. This further indicates the robustness of our applied methods for CSA measurements in 1.5-T MRI, using semi-automated for $\mathrm{CT}$ and manual delineation for MRI to determine sarcopenic patients.

However, some limitations have to be mentioned. External validation of our findings could be considered the major limitation of this present study. This is due to missing cross-validation of the formula of Swartz et al to calculate CSA at the L3 level through CSA at the C3 level, and the lack of large-scale validated SMI cut-off values for sarcopenia detection in head and neck cancer 

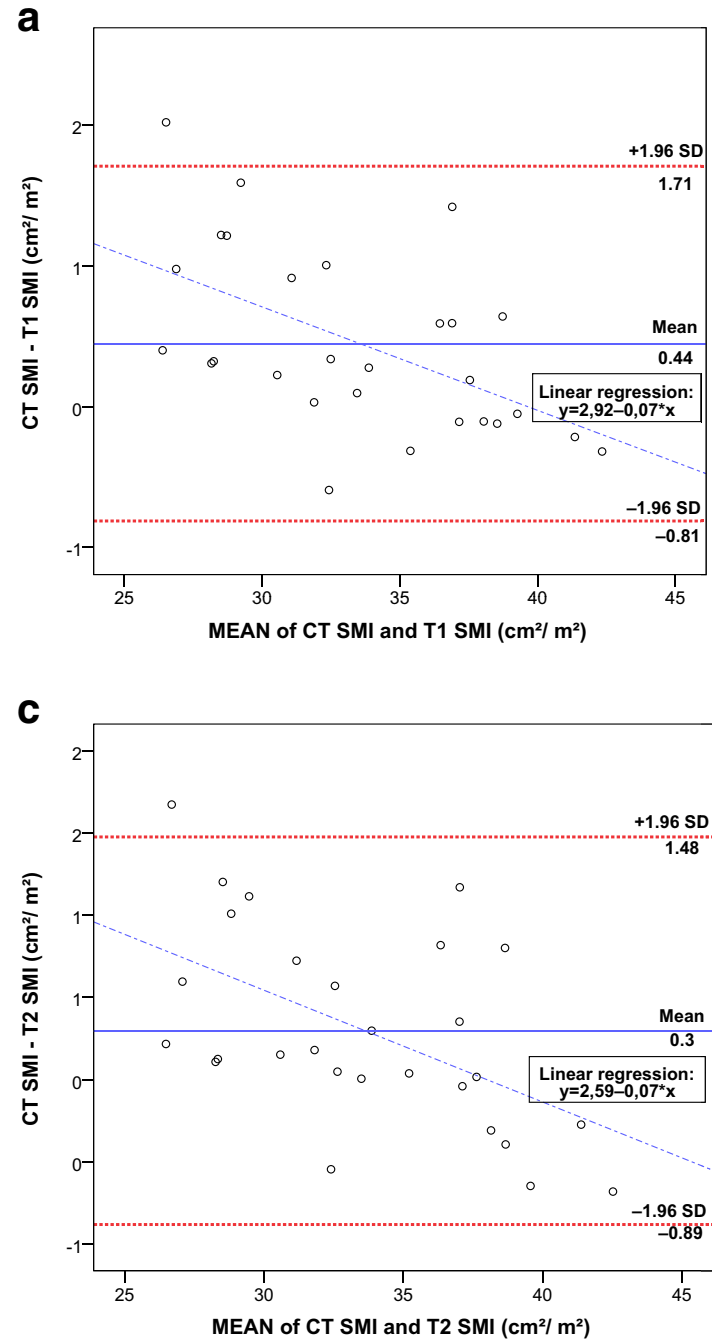

Fig. 4 Bland-Altman plots of mean SMI and $\triangle$ SMI between CT and 1.5-T MRI stratified for gender. Boundaries with the $95 \%$ confidence interval ( \pm 1.96 times the standard deviation) are given for the mean $\Delta \mathrm{SMI}$ and linear regression analysis, except for $\mathbf{d}$. For women $(n=$

patients $[7,8]$. However, a recent published study found a strong and significant correlation between SMI determined at the C3 and L3 level [27]. Further research should therefore be focussed on cross-validation of the formula of Swartz et al and head and neck cancer patient-specific SMI cut-off values for determining sarcopenia in larger cohorts. We applied the gender independent SMI cut-off value of Wendrich et al as it has a very similar head and neck cancer population. In previous studies, the use of gender-dependent cut-off values was proposed [7, 28], but also seen as a difficult task as women have a smaller ratio within the head and neck cancer population. Furthermore, qualitative research with muscle CT radio-density should be validated, as recently muscle density was reported to be more associated with frailty in older adults with cancer, than skeletal muscle area [29]. CSA
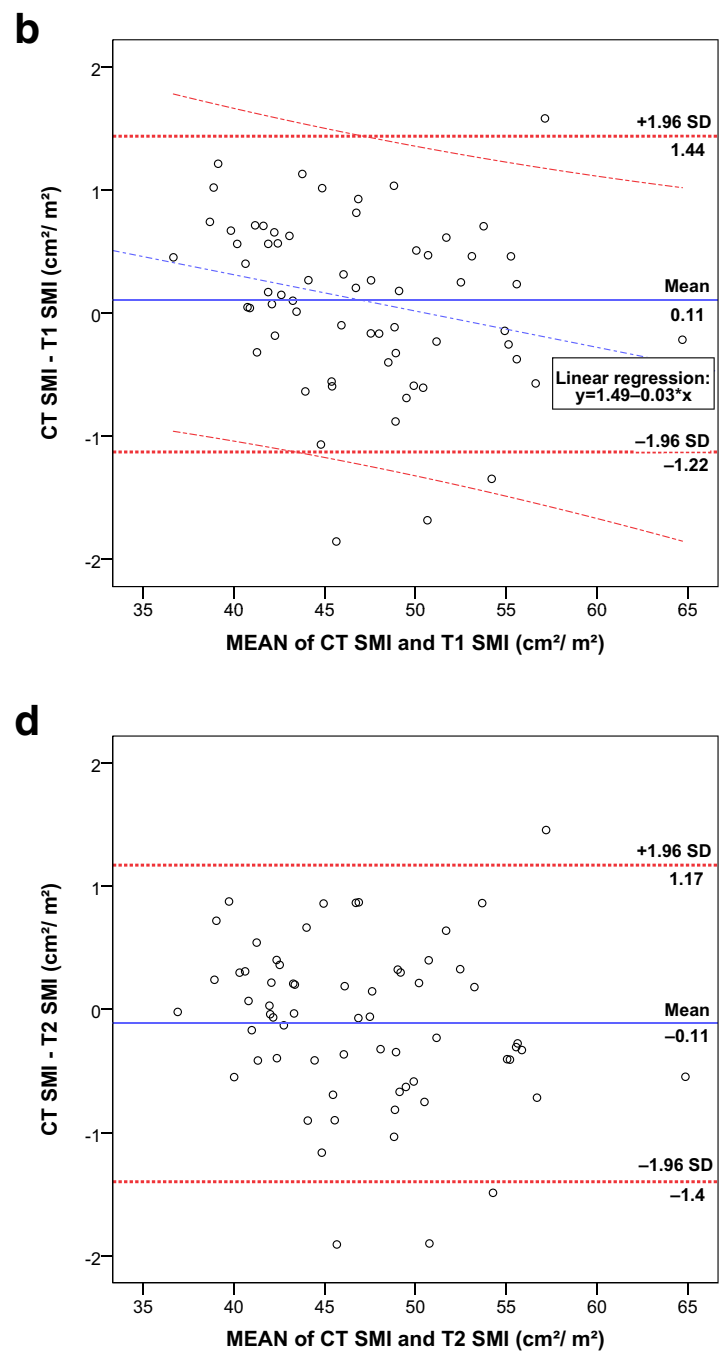

28), CT SMI versus T1 SMI (a) and CT SMI versus T2 SMI (c); and for men $(n=64)$, CT SMI versus T1 SMI (b) and CT SMI versus T2 SMI (d). SMI skeletal muscle index, $C T$ computed tomography, $M R I$ magnetic resonance imaging

measurements should be validated on low-dose CT neck scans to ensure that all head and neck cancer patients can be screened for sarcopenia before, during, and after treatment. Our study has nevertheless multiple strengths. Firstly, the study was performed in a relatively large group of patients from a prospectively maintained data-biobank. Secondly, an excellent inter- and intraobserver agreement by three observers was demonstrated in the random reselected neck $\mathrm{CT}$ and MRI scans, proving that CSA measurements at the level of $\mathrm{C} 3$ are both reproducible and reliable. Thirdly, high grades can be given for the short time interval between CT and MRI minimizing the impact of pathophysiological muscle change to an absolute minimum. Furthermore, data was stratified for gender as previous studies emphasized that SMI differences exist between men and women. 
Table 4 Sarcopenia categorization between $\mathrm{CT}$ and MRI

\begin{tabular}{lllll}
\hline & SMI $<43.2 \mathrm{~cm}^{2} / \mathrm{m}^{2}$ & $\mathrm{SMI} \geq 43.2 \mathrm{~cm}^{2} / \mathrm{m}^{2}$ & Cohen's $\kappa(95 \% \mathrm{CI})$ & McNemar \\
\hline 1.5-T & & & & \\
CT SMI & $n=47$ & $n=45$ & & $p$ value \\
T1 SMI & $49(+4 \%)$ & $43(-4 \%)$ & $0.956(0.897-1)^{*}$ & 0.500 \\
T1 vibe SMI & $46(-2 \%)$ & $46(+2 \%)$ & $0.978(0.936-1)^{*}$ & 1.000 \\
T2 SMI & $48(+2 \%)$ & $46(-2 \%)$ & $0.978(0.936-1)^{*}$ & 1.000 \\
3-T & & & & \\
CT SMI & $n=11$ & $n=22$ & & $0.870(0.725-1)^{*}$ \\
T1 SMI & $13(+18 \%)$ & $20(-9 \%)$ & $0.870(0.725-1)^{*}$ & 0.500 \\
T1 contrast SMI & $13(+18 \%)$ & $20(-9 \%)$ & $0.870(0.725-1)^{*}$ & 0.500 \\
T1 DIXON SMI & $13(+18 \%)$ & $20(-9 \%)$ & $0.933(0.827-1)^{*}$ & 1.000 \\
T2 DIXON SMI & $12(+9 \%)$ & $21(-4 \%)$ & & \\
\hline
\end{tabular}

Relative difference of sarcopenia diagnosis for both 1.5-T $(n=92)$ and 3-T $(n=33) M R I$ sequences respective to $\mathrm{CT}$, with agreement and differences of sarcopenia categorization between the two modalities analyzed with Cohen's kappa coefficient and McNemar test, respectively. SMI skeletal muscle index, $C I$ confidence interval. *Significant $p$ value $<0.001$

In conclusion, CSA measurements on CT and 1.5-T and 3$\mathrm{T}$ MRI neck scans at the $\mathrm{C} 3$ level can be used interchangeably. In the event no neck $\mathrm{CT}$ scan is performed, skeletal muscle mass and radiological sarcopenia can be determined with CSA measurements on neck MRI scans. This finding contributes to the construction of a clinical useful radiological biomarker for measuring radiological sarcopenia in head and neck cancer patients, which has previously been emphasized by others $[18,19]$.

Supplementary Information The online version contains supplementary material available at https://doi.org/10.1007/s00330-020-07440-1.

Funding The first author was awarded with a 3-years-PhD scholarship for excellent master students from the Graduate School of Medical Sciences of the University of Groningen.

\section{Compliance with ethical standards}

Guarantor The scientific guarantor of this publication is A. van der Hoorn.

Conflict of interest The authors of this manuscript declare no relationships with any companies whose products or services may be related to the subject matter of the article.

Statistics and biometry One of the authors has significant statistical expertise.

Informed consent Written informed consent was obtained from all subjects (patients) by OncoLifeS in this study.

Ethical approval OncoLifeS is a large oncological data-biobank which is approved by the Medical Ethical Committee of the University Medical Centre of Groningen. The scientific board of OncoLifeS gave permission to use the data for this present study.
Study subjects or cohorts overlap In this current manuscript, data of 50 of the 125 patients have been reported previously by Zwart et al in Zwart AT, van der Hoorn A, van Ooijen, P M A, Steenbakkers, R J H M, de Bock GH, Halmos GB (2019) "CT-measured skeletal muscle mass used to assess frailty in patients with head and neck cancer." J Cachexia Sarcopenia Muscle. In the previously published study, we quantified pre-treatment skeletal muscle mass on neck CT images and no neck MRI images were used. None of the MRI measurements has been published earlier.

\section{Methodology}

- Retrospective analysis on prospectively included data

- Cross-sectional study

- Performed at one institution

Open Access This article is licensed under a Creative Commons Attribution 4.0 International License, which permits use, sharing, adaptation, distribution and reproduction in any medium or format, as long as you give appropriate credit to the original author(s) and the source, provide a link to the Creative Commons licence, and indicate if changes were made. The images or other third party material in this article are included in the article's Creative Commons licence, unless indicated otherwise in a credit line to the material. If material is not included in the article's Creative Commons licence and your intended use is not permitted by statutory regulation or exceeds the permitted use, you will need to obtain permission directly from the copyright holder. To view a copy of this licence, visit http://creativecommons.org/licenses/by/4.0/.

\section{References}

1. Gatta G, Botta L, Sanchez MJ et al (2015) Prognoses and improvement for head and neck cancers diagnosed in Europe in early 2000s: the EUROCARE-5 population-based study. Eur J Cancer 51:2130 2143

2. Dutch Cancer Registration. [Internet]; c2019 [cited 2019 06-08]. Available from: https://www.cijfersoverkanker.nl/selecties/ Dataset_1/img5d495e2aa4a7e 
3. Cruz-Jentoft AJ, Bahat G, Bauer J et al (2019) Sarcopenia: revised European consensus on definition and diagnosis. Age Ageing 48: 16-31. https://doi.org/10.1093/ageing/afy169

4. Zwart AT, van der Hoorn A, van Ooijen PMA, Steenbakkers RJHM, de Bock GH, Halmos GB (2019) CT-measured skeletal muscle mass used to assess frailty in patients with head and neck cancer. J Cachexia Sarcopenia Muscle. https://doi.org/10.1002/ jesm. 12443

5. Hua X, Liu S, Liao JF et al (2020) When the loss costs too much: a systematic review and meta-analysis of sarcopenia in head and neck cancer. Front Oncol 9:1561. https://doi.org/10.3389/fonc.2019. 01561

6. Findlay M, White K, Lai M, Luo D, Bauer JD (2020) The association between computed tomography-defined sarcopenia and outcomes in adult patients undergoing radiotherapy of curative intent for head and neck cancer: a systematic review. J Acad Nutr Diet 120:1330-1347.e8

7. Wendrich AW, Swartz JE, Bril SI et al (2017) Low skeletal muscle mass is a predictive factor for chemotherapy dose-limiting toxicity in patients with locally advanced head and neck cancer. Oral Oncol 71:26-33

8. Swartz JE, Pothen AJ, Wegner I et al (2016) Feasibility of using head and neck CT imaging to assess skeletal muscle mass in head and neck cancer patients. Oral Oncol 62:28-33

9. Lewis-Jones H, Colley S, Gibson D (2016) Imaging in head and neck cancer: United Kingdom national multidisciplinary guidelines. J Laryngol Otol 130:S28-S31

10. Chung NN, Ting LL, Hsu WC, Lui LT, Wang PM (2004) Impact of magnetic resonance imaging versus $\mathrm{CT}$ on nasopharyngeal carcinoma: primary tumor target delineation for radiotherapy. Head Neck 26:241-246. https://doi.org/10.1002/hed.10378

11. King AD, Teo P, Lam WW, Leung SF, Metreweli C (2000) Paranasopharyngeal space involvement in nasopharyngeal cancer: detection by CT and MRI. Clin Oncol (R Coll Radiol) 12:397-402

12. Poon PY, Tsang VH, Munk PL (2000) Tumour extent and T stage of nasopharyngeal carcinoma: a comparison of magnetic resonance imaging and computed tomographic findings. Can Assoc Radiol J 51:287-295

13. Mitsiopoulos N, Baumgartner RN, Heymsfield SB, Lyons W, Gallagher D, Ross R (1998) Cadaver validation of skeletal muscle measurement by magnetic resonance imaging and computerized tomography. J Appl Physiol (1985) 85:115-122

14. Engstrom CM, Loeb GE, Reid JG, Forrest WJ, Avruch L (1991) Morphometry of the human thigh muscles. A comparison between anatomical sections and computer tomographic and magnetic resonance images. J Anat 176:139-156

15. Faron A, Sprinkart AM, Kuetting DLR et al (2020) Body composition analysis using CT and MRI: intra-individual intermodal comparison of muscle mass and myosteatosis. Sci Rep 10:11765. https://doi.org/10.1038/s41598-020-68797-3

16. Khil EK, Choi JA, Hwang E, Sidek S, Choi I (2020) Paraspinal back muscles in asymptomatic volunteers: quantitative and qualitative analysis using computed tomography (CT) and magnetic resonance imaging (MRI). BMC Musculoskelet Disord 21:403. https:// doi.org/10.1186/s12891-020-03432-w
17. Sinelnikov A, Qu C, Fetzer DT et al (2016) Measurement of skeletal muscle area: comparison of CT and MR imaging. Eur J Radiol $85: 1716-1721$

18. Chargi N, Ansari E, Huiskamp LFJ, Bol G, de Bree R (2019) Agreement between skeletal muscle mass measurements using computed tomography imaging and magnetic resonance imaging in head and neck cancer patients. Oral Oncol 99:104341. https:// doi.org/10.1016/j.oraloncology.2019.06.022

19. Bril SI, Wendrich AW, Swartz JE et al (2019) Interobserver agreement of skeletal muscle mass measurement on head and neck CT imaging at the level of the third cervical vertebra. Eur Arch Otorhinolaryngol 276:1175-1182. https://doi.org/10.1007/s00405019-05307-w

20. Sidorenkov G, Nagel J, Meijer C et al (2019) The OncoLifeS databiobank for oncology: a comprehensive repository of clinical data, biological samples, and the patient's perspective. J Transl Med 17: 374. https://doi.org/10.1186/s12967-019-2122-x

21. Mourtzakis M, Prado CM, Lieffers JR, Reiman T, McCargar LJ, Baracos VE (2008) A practical and precise approach to quantification of body composition in cancer patients using computed tomography images acquired during routine care. Appl Physiol Nutr Metab 33:997-1006. https://doi.org/10.1139/H08-075

22. McNEMAR Q (1947) Note on the sampling error of the difference between correlated proportions or percentages. Psychometrika 12: $153-157$

23. Landis JR, Koch GG (1977) The measurement of observer agreement for categorical data. Biometrics 33:159-174

24. Smith AC, Parrish TB, Abbott R et al (2014) Muscle-fat MRI: 1.5 tesla and 3.0 tesla versus histology. Muscle Nerve 50:170-176. https://doi.org/10.1002/mus.24255

25. Gotra A, Chartrand G, Massicotte-Tisluck K et al (2015) Validation of a semiautomated liver segmentation method using CT for accurate volumetry. Acad Radiol 22:1088-1098. https://doi.org/10. 1016/j.acra.2015.03.010

26. Ghatas MP, Lester RM, Khan MR, Gorgey AS (2018) Semiautomated segmentation of magnetic resonance images for thigh skeletal muscle and fat using threshold technique after spinal cord injury. Neural Regen Res 13:1787-1795. https://doi.org/10.4103/ 1673-5374.238623

27. Ufuk F, Herek D, Yuksel D (2019) Diagnosis of sarcopenia in head and neck computed tomography: cervical muscle mass as a strong indicator of sarcopenia. Clin Exp Otorhinolaryngol 12:317-324. https://doi.org/10.21053/ceo.2018.01613

28. Bril SI, Pezier TF, Tijink BM, Janssen LM, Braunius WW, de Bree R (2019) Preoperative low skeletal muscle mass as a risk factor for pharyngocutaneous fistula and decreased overall survival in patients undergoing total laryngectomy. Head Neck 41:1745-1755. https://doi.org/10.1002/hed.25638

29. Williams GR, Deal AM, Muss HB et al (2018) Frailty and skeletal muscle in older adults with cancer. J Geriatr Oncol 9:68-73

Publisher's note Springer Nature remains neutral with regard to jurisdictional claims in published maps and institutional affiliations. 